\title{
Equation of state with scale-invariant hidden local symmetry and gravitational waves
}

\author{
Hyun Kyu Lee ${ }^{1, \star}$ \\ ${ }^{1}$ Department of Physics, Hanyang University, Seoul 04763, Korea
}

\begin{abstract}
The equation of state (EoS) for the effective theory proposed recently in the frame work of the scale-invariant hidden local symmetry is discussed briefly. The EoS is found to be relatively stiffer at lower density and but relatively softer at higher density. The particular features of EoS on the gravitational waves are discussed. A relatively stiffer EoS for the neutron stars with the lower density induces a larger deviation of the gravitational wave form from the point-particle-approximation. On the other hand, a relatively softer EoS for the merger remnant of the higher density inside might invoke a possibility of the immediate formation of a black hole for short gamma ray bursts or the appearance of the higher peak frequency for gravitational waves from remnant oscillations. It is anticipated that this particular features could be probed in detail by the detections of gravitational waves from the binary neutron star mergers.
\end{abstract}

\section{Introduction}

The study of QCD under extreme conditions at high temperature and/or at high baryon number density has been one of the leading research subjects in understanding the theory of strong interaction. The object of this study includes the early Universe at high temperature as well as the core of the compact object with high density. While there has been lots of interesting progress in understanding QCD at high temperature theoretically and experimentally, at RHIC and LHC for example, there has been little development in QCD at high density and it remains as an uncharted area.

There are several experimental projects proposed or being constructed in near future like FAIR (Germany), FRIB (USA), HIAF (China), NICA (Russia), RAON (Korea), RIBF (Japan) as facilities for exploring the dense hadronic matter. The target density depending on the facilities is expected to be more than 2 times (but not much higher) normal nuclear density, $2 n_{0}$. Neutron stars are believed to be compact objects with high density cores inside. The core densities of typical neutron stars of mass up to $1.5 M_{\odot}$ is estimated to be roughly $2-3 n_{0}$. On the other hand the core densities of the recently observed two-solar-mass neutron stars [1] are believed to be most likely greater than $5 n_{0}$.

Recent detections of gravitational waves form black hole binaries [2] indicate strongly the possibilities of detecting gravitational waves from coalescing binary-neutron stars (BNS). According to the population analysis based on the designed detection range of BNS gravitational waves of aLIGO $\sim 100 \mathrm{Mpc}$, a number of detections of gravitational waves from BNSs are expected in $\mathrm{O} 2$ and $\mathrm{O} 3$ runs at aLIGO and aVirgo[3].

\footnotetext{
^e-mail: hyunkyu@hanyang.ac.kr
} 
For a static and spherically symmetric astrophysical compact star, the relativistic hydrodynamic equilibrium is governed by the Tolmann-Oppenheimer-Volkoff(TOV) equation

$$
\frac{d m}{d r}=4 \pi r^{2} \epsilon, \frac{d p}{d r}=-(\epsilon+p) \frac{m+4 \pi r^{3} p}{r(r-2 m)}, \frac{d \Phi}{d r}=-\frac{1}{\epsilon+p} \frac{d p}{d r} .
$$

where $\epsilon$ and $p$ are energy density and pressure at $r$ respectively. $m(r)$ is the mass enclosed inside the radius $r$. We can calculate the mass of compact star, $M$, and its radius, $R$, by integrating the TOV equation up to $p(R)=0$ and we get the profile of $\Phi(r), m(r)$ and $p(r)$.

When a compact star is placed in a static external field, a star supposed to be in a spherically symmetric configuration is then deformed by the external field. The asymptotic expansion of the metric at large distances $r$ from the star defines the quadrupole moment, $Q_{i j}$, and the external tidal field, $\mathcal{E}_{i j}$, as expansion coefficients [4] given by $-\frac{1+g_{00}}{2}=-\left[\frac{m}{r}+\frac{3}{2} \frac{Q_{i j}}{r^{3}} n^{i} n^{j}+\cdots\right]+\frac{1}{2} \mathcal{E}_{i j} r^{2} n^{i} n^{j}+\cdots$ where $n^{i}=x^{i} / r$ and $Q_{i j}$ and $\mathcal{E}_{i j}$ are both symmetric and traceless. The deformability parameter $\lambda$ is defined by

$$
Q_{i j}=-\lambda \mathcal{E}_{i j} \text { or } \Lambda=\lambda G\left(\frac{c^{2}}{G M}\right)^{5}=9.51 \times 10^{2} \lambda_{36}\left(\frac{M_{\odot}}{M}\right)^{5}
$$

where $G$ is the gravitational constant and $\lambda=\lambda_{36} \times 10^{36} \mathrm{~g} \mathrm{~cm}^{2} \mathrm{~s}^{2}$. Deformation parameters ${ }^{1}$ obtained from the known EoSs consistent with certain criteria, for example on velocity of sound $v_{s}<1$ and on maximum mass $M_{\text {max }} \geq 2 M_{\odot}$, are calculated to be in a rather broad range of $10^{2}-10^{3}[5,7]$.

The effect of the tidal deformation on the gravitational waves in binary coalescence process is found to be appearing at the order of post newtonian approximation in the analytical formulation. The numerical simulation matched to the analytic expression shows a clear deviation from point particle approximation at high frequency (up to near $1 \mathrm{kHz}$ ) component of the gravitational wave: the larger tidal deformability $\Lambda$, the more deviation. It also shows the feasibility of distinguishability between EoSs if $\delta \Lambda>100$ [8]. At the final stage of the binary coalescing process, there are two possibilities depending on the EoS, a prompt emergence of a black hole for soft EoSs or a merger remnant for sufficiently stiffer EoSs. For a sufficiently stiffer EoS, for which prompt collapse into a black hole is unlikely, the results of numerical simulations demonstrate interesting results of the appearance of peaks in their higher frequency components, $f>1 \mathrm{kHz}$ [9]. The peaks in the frequency components are considered to be the evidence of the gravitational emission during the remnant oscillations. The location of peaks, $f_{2}$, due to the quadrupolar oscillation are found to be sensitive on the EoS such that a larger deformation $\Lambda$ leads to a smaller peak frequency $f_{2}$.

Recently a new EoS has been proposed in the quite different frame work [10,11] when compared with the presently available EoSs. The EoS is obtained based on the model constructed in responding to the question whether certain fundamental symmetries of QCD, invisible or hidden in matter-free space, can emerge at high baryonic density such as in massive compact stars. Therefore it is very interesting to see whether its particular characteristics can be probed by gravitational waves, which will be discussed briefly in the final section.

\section{EoS with scale-invariant hidden local symmetry}

The constituents of a neutron stars are mostly neutrons with a finite fraction of protons and electrons, for low mass neutron stars with core density not much higher than $2 n_{0}$. In recent papers [11], it was proposed that both hidden local symmetry and hidden scale symmetry could emerge at densities

\footnotetext{
${ }^{1}$ For details how to obtain the deformation parameters in combination with TOV, see for example [5, 6].
} 
relevant to $\sim 2$ solar-mass neutron stars. As density increases it is natural to expect new degrees of freedom to be exited with the explicit emergence of those symmetries. The particle contents entangled with hidden local symmetry are rho meson and omega meson and a scalar particle, dilaton $\chi$ as a conformal compensator with scale symmetry. The corresponding lagrangian, $\mathcal{L}_{\text {inv }}$, dictated by the symmetries with particle contents can be written down in a straightforward way [11]. The only term which does not obey the scale symmetry, $\mathcal{L}_{\mathrm{SB}}$, is the dilaton potential term, $V(\chi)$, which is put such that it produce the QCD scale anomaly properly.

$$
\theta_{\mu}^{\mu}=4 V(\chi)-\chi \frac{\partial V(\chi)}{\partial \chi}\left(=\frac{\beta\left(\alpha_{s}\right)}{4 \alpha_{s}} G_{\mu \nu}^{a} G^{a \mu \nu}\right)
$$

where $G^{\mu v}$ is the gluon field in QCD.

In the medium characterized by density $n$, the basic premise is that the effect of changes in the vacuum structure caused by density is entirely encoded in the density dependent parameters of the Lagrangian up to the density domain relevant in compact stars. How these density dependencies, intrinsic density dependencies (IDDs), are defined is described in detail in [10]. For the refinement of the IDDs behavior, the sophisticated high order correlation beyond the mean field treatment, the $V_{\text {lowk }}-\mathrm{RG}$ treatment is adopted.

Up to nuclear matter density $n_{0} \simeq 0.16 \mathrm{fm}^{-3}$, IDDs can be more or less determined either from experiments or theoretically. It will however be extremely difficult to track them at higher densities going beyond $n_{0}$. Here we rely on certain topological structure present in the skyrmion approach. An extremely important observation in the skyrmion approach, when skyrmions are put on crystal lattice, is the changeover from skyrmions to half-skyrmions at a density $n>n_{0}$ [12]. When the topology change is translated into the bare parameters, it makes a drastic - and novel - change in the IDDs across the transition density denoted $n_{1 / 2}$. Specifically it gives the prediction that going into the halfskyrmion phase, the in-medium pion (dilaton) decay constant $f_{\pi}^{*}\left(f_{\sigma}^{*}\right)$ and the in-medium nucleon mass $m_{N}^{*}$ go over to a constant $f_{\pi}^{*} / f_{\pi} \approx f_{\sigma}^{*} / f_{\sigma} \approx m_{N}^{*} / m_{N} \rightarrow \kappa \sim(0.6-0.9)$.

At the lower density $n \leq n_{1 / 2}$, the results of the $V_{\text {lowk }}$ calculation for $n \lesssim n_{1 / 2}$ can be well fit for the neutron matter by the fitting functions for energy density and pressure given by

$$
\epsilon=m_{N} n+n_{0} A_{I}\left(\frac{n}{n_{0}}\right)^{2}+n_{0} B_{I}\left(\frac{n}{n_{0}}\right)^{1+D_{I}}, p=n_{0} A_{I}\left(\frac{n}{n_{0}}\right)^{2}+n_{0} B D_{I}\left(\frac{n}{n_{0}}\right)^{D_{I}+1} .
$$

where $\left(A_{I}, B_{I}, D_{I}\right)=(9.11 \mathrm{MeV}, 2.14 \mathrm{MeV}, 4.08)$.

In the density regime $n>n_{1 / 2}$, there is a topology change that drastically affects the IDDs at $n=n_{1 / 2}$, parameters changed from those in $n \leq n_{1 / 2}$ as explained in detail in [11]. It turns out that the results of the $V_{\text {lowk }}$ calculation for $n>n_{1 / 2}$ can be captured very well by the simple formula

$$
\epsilon=n_{0} B\left(\frac{n}{n_{0}}\right)^{4 / 3}+n_{0} D, \quad p=\frac{1}{3} n_{0} B\left(\frac{n}{n_{0}}\right)^{4 / 3}-n_{0} D,
$$

where $B=686 \mathrm{MeV}, D=253 \mathrm{MeV}$ for the pure neutron matter.

It is interesting to note that the equation of state looks very similar to the relativistic free gas with a 'bag-constant-like'term $n_{0} D\left(\sim 41 \mathrm{MeV} / \mathrm{fm}^{3}\right.$ for the pure neutron matter $)$. The former is consistent with the conformal symmetric nature of the effective theory considered here and the latter the scalesymmetry-breaking dilaton potential. The observation that the 'bag-constant-like' is found to be a constant independent on the density leads to the conclusion that the velocity of sound is given by the conformal value, $\sqrt{1 / 3} c$.

Given the equation of state described above, it is a standard procedure to use the TOV equation to calculate the properties of compact stars. In doing this we will take into account of the $\beta$ stability, 
thereby including the leptons. The maximum mass of compact star is estimated to be $\simeq 2.05 M_{\odot}$ and the radius $R \simeq 12.19 \mathrm{~km}$ with the maximum central density $n_{\max } \simeq 5.1 n_{0}$. The result is consistent with the well-measured values $M \sim 2 M_{\odot}$ [1]. It is fair to conclude that the effective theory provides an evidently respectable EoS for massive compact stars. Up to the predicted central density $\sim 5.1 n_{0}$, there are no other degrees of freedom than the pseudo-NG bosons ( $\pi$ and $\sigma$ ), vector mesons and nucleons.

\section{Discussion}

A simple comparison with other EoSs in the literature can be given using polytropic fits. The adiabatic indices, $\Gamma \mathrm{s}$, for polytrope fits of EoSs [13] are compared with the highest indices in Eqs.4 and 5. At the lower density $n \leq n_{1 / 2}$, the highest index, $\sim 5$, in Eq.4 is found to be larger than adiabatic indices Гs. At the higher density $n>n_{1 / 2}$ the highest index in Eq. 5 is fund to be $4 / 3$, which is less than those in [13].

During the early phase of coalescing process neutron stars in binary can be treated as point particles as far as gravitational wave is concerned and the gravitational wave depends essentially on their masses of neutron stars such that there is no more information on equation of states than the masses. Since the most abundant NSs are believed to have masses around $1.4 M_{\odot}$, the density to be explored is not expected to be much higher than $\sim 2 n_{0}$ [14]. When they get closer the gravity between neutron stars becomes strong enough to induce tidal deformations on each orbiting neutron star. The point particle approximation is no more valid and the effect of tidal deformation on the gravitational wave should be considered. The tidal deformation depends strongly on the equation of states and therefore the deviation of gravitational waves form the point particle approximation will certainly provide an useful information in differentiating various EoSs, which otherwise cannot be distinguished by the masses alone. At the final stage of merging process, there is a possibility of appearing a black hole or a merger remnant if not prompt collapse into a black hole, which depends on the EoS of merging neutron stars. Numerical simulation demonstrates that the density of the remnants can be as large as more than $\sim 4 n_{0}$ even for the merging neutrons stars of masses, $1.4 M_{\odot}$.

For $1.4 M_{\odot}$ neutron star with core density $2 n_{0}$ with new EoS, the tidal deformation parameter is calculated to be $\lambda_{36}=4.44$ or $\Lambda=815$, which is larger than the most of the other known EoSs for $1.4 M_{\odot}$ neutron star. It is because the EoS is stiffer with larger deformation parameter for the density of the $1.4 M_{\odot}$ star. And consequently the larger deviation from point-like-approximation in the gravitational wave form from the coalescing binary is expected. On the other hand, the density of the merger remnants are expected to be higher than $\sim 4 n_{0}$, at which the new EoS is found to become softer. Hence the prompt formation of a black hole after merger might be one of the interesting expectations. The possibility could be explored in numerical simulation when the change over of the EoS from lower density relevant to binary neutron stars into higher density relevant for a remnant is seriously considered. The viable scenario of soft gamma ray bursts is the presence of a black hole at the central region. When the black hole for short GRBs is formed from the binary coalescence, then softer EoS at higher density might be preferred for those GRBs followed by gravitational waves. If it is not soft enough to form a prompt black hole, an oscillating merger remnant might be expected with the peak frequency feature. But the peak frequency for new EoS is likely to be higher than those of stiffer EoSs [9].

In summary, it is observed that the EoS with scale-invariant hidden local symmetry behaves as a stiffer EoS in a sense that it gives a larger radius and larger deformation parameter, which in turn results in the larger deviation of gravitational wave from point-like-approximation. In the higher density regime for the merger remnant it behaves as relatively softer EoS with the possibility of a prompt formation of a black hole for short gamma ray bursts or higher peak frequency for gravitational 
waves from remnant oscillations. These particular features could be probed in detail by the detections of gravitational waves from the binary neutron star mergers.

The author would like to thank Kyungmin Kim, Tom Kuo, Yong-Liang Ma, Won-Gi Paeng and Mannque Rho for the useful discussions and long-time collaborations. This work was supported in part by a Collaborative Research Grant from KISTI GSDC in 2017.

\section{References}

[1] P.B. Demorest et al., Nature 467, 1081 (2010); J. Antoniadis et al., Science 340, 6131 (2013).

[2] B.P. Abbot et al. , Phys. Rev. Lett. 116, 061102 (2016).

[3] J. Aasi, et al., Class. Quantum Grav. 32, 074001 (2015);F. Acernese, et al., Class. Quantum Grav. 32(2), 024001 (2015).

[4] K. S.Thorne, Phys. Rev. D 58, 124031 (1998)

[5] T. Hinderer, B.D. Lackey, R.N. Lang and J.S. Read, Phys. Rev. D 81, 123016 (2010).

[6] K. Kim, H.K. Lee and J. Lee, Int. J. Mod. Phys. E 26, 1740011 (2017)

[7] J.S. Read et al., Phys. Rev. D 88, 044042 (2013)

[8] K. Hotokezaka, K. Kyutoku, Y. Sekiguchi and M. Shibata, Phys. Rev. D 93, 064082 (2016).

[9] A. Bauswein, N.Stergioulas, H-T. Janka, EPJA 52, 56B(2016)

[10] W.G. Paeng, T.T.S. Kuo, H.K. Lee and M. Rho, Phys. Rev. C 93, 055203 (2016).

[11] W.G. Paeng, T.T.S. Kuo, H. K. Lee, Yong-Liang Ma and M. Rho, Phys. Rev. D 96, 014031 (2017).

[12] H.K. Lee, B.Y. Park and M. Rho, Phys. Rev. C 83, 025206 (2011) Erratum: [Phys. Rev. C 84, 059902 (2011)].

[13] J.S. Read, B.D. Lackey, B.J. Owen, J.L. Friedman, Phys. Rev. D79, 124032(2009).

[14] J. Lattimer, Ann. Rev. Nucl. Part. Sci. 62, 485(2012). 